Artigo / Article

\title{
Tratamento de primeira linha no Mieloma Múltiplo
}

\section{First line treatment in Multiple Myeloma}

Gisele W. B. Colleoni

\section{Introdução}

Devido à heterogeneidade das alterações genéticomoleculares envolvidas na sua fisiopatologia, o mieloma múltiplo (MM) é uma doença extremamente variável do ponto de vista de apresentação clínica e evolução, sendo a sobrevida mediana dos pacientes de aproximadamente três anos a partir do diagnóstico. ${ }^{1}$

A presente revisão tem por objetivo avaliar o estado atual do tratamento de primeira linha dos pacientes com MM.

Para isso, realizamos revisão da literatura e apresentamos o resultado de estudos clínicos randomizados, guidelines de sociedades científicas ou centros médicos internacionais de reconhecida importância, informações obtidas em eventos científicos recentes e experiência pes- soal de especialistas brasileiros, especialmente convidados para integrar esse consenso.

\section{Quando tratar o paciente com diagnóstico recente de MM?}

Utilizando os critérios recomendados pelo International Myeloma Working Group, ${ }^{2}$ os pacientes com MM são classificados como: assintomáticos ou sintomáticos. Apesar dos avanços no tratamento do MM nos últimos anos, não existem evidências, até o momento, de que o MM assintomático (também conhecido como MM smoldering) deva receber tratamento ao diagnóstico. Nestes pacientes a mediana de progressão para doença sintomática é de dois a três anos, havendo tempo para indicar tratamento quando surgirem os sintomas. ${ }^{3}$

Professora adjunta da Disciplina de Hematologia e Hemoterapia - Universidade Federal de São Paulo, Unifesp/EPM.

Correspondência: Gisele W. B. Colleoni

Rua Botucatu, 740, $3^{\circ}$ andar - Hematologia

04023-900 - São Paulo-SP - Brasil

Tel.: (11) 5579-1550; Fax: (11)5571-8806

E-mail: gcolleoni@hemato.epm.br 


\section{Quais as possibilidades de tratamento de primeira linha para o MM?}

Para o paciente sintomático (anemia, hipercalcemia, alteração da função renal, presença de lesões líticas ou plasmocitoma extramedular, aumento progressivo do componente-M no soro e/ou urina), o tratamento deverá ser rapidamente instituído. Nesse caso, temos dois grandes grupos de pacientes: aqueles que são considerados candidatos à consolidação com altas doses de quimioterapia seguidas de transplante de células-tronco hematopoéticas e um esquema $\mathrm{VAD}^{4}$ não parece ser melhor do que dexametasona isoladamente (taxas de resposta em casos recém-diagnosticados de 55\%-65\% e 45\%, respectivamente) e esse último parece ser o agente realmente ativo no VAD. Além disso, a dexametasona apresenta como vantagens o baixo risco de alopécia ou infecção, apesar de desencadear quadros de hiperglicemia principalmente na população de pacientes mais idosos. $^{5-8}$

O VAD foi inicialmente concebido como um esquema de quimioterapia em infusão contínua, considerando que essa poderia ser uma alternativa para superar a resistência tumoral às drogas. Do ponto de vista prático, quatro dias de internação para receber medicamentos em infusão contínua por quatro a seis ciclos pode ser inviável na maioria dos hospitais públicos brasileiros. Por isso, desde 2001, temos utilizado na Unifesp o esquema VAD ambulatorial, com infusão em trinta minutos da vincristina e doxorubicina. Esse esquema, proposto por Segeren et al, ${ }^{9}$ do Dutch-Belgian HematologyOncology Cooperative Group (HOVON), ainda prevê uso de dexametasona 40 mg VO/dia D1-4, D9-12, D17-20 nos ciclos ímpares e D1-4 nos ciclos pares. Apesar desse estudo não ser randomizado, o VAD ambulatorial garantiu taxa de resposta de $67 \%$ no grupo de pacientes com diagnóstico recente de MM, sendo esta semelhante ao VAD clássico. Em nossa experiência, a redução da dose de dexame-

Figura 1. Proposta para tratamento de pacientes com $\mathrm{MM}^{1}$

segundo grupo de pacientes não elegíveis para receber altas doses de quimioterapia (Figura 1 ). ${ }^{1}$ Os critérios utilizados para alocar os pacientes em um desses grupos baseiam-se em idade, performance status e presença de comorbidades.

\section{Pacientes com menos de 65-70 anos, com bom performance status, sem comorbidades, com função renal próxima do normal}

Estes indivíduos são potencialmente candidatos à consolidação com altas doses de quimioterapia seguidas de transplante autólogo de células tronco-hematopoéticas. A coleta das células-tronco deve ser feita antes do uso de agentes alquilantes, os quais potencialmente prejudicam a mobilização destas células e a recuperação hematopoética pós-transplante.

Portanto, para pacientes com indicação de transplante autólogo, são preferíveis regimes de quimioterapia como blocos de dexametasona, VAD (vincristina, doxorubicina e dexametasona) ou talidomida \pm dexametasona (Quadro 1). O tasona nos ciclos pares diminuiu consideravelmente a incidência de diabetes e o número de infecções pulmonares, aparentemente sem prejuízo nas taxas de resposta em relação ao VAD em infusão contínua.

Estudos clínicos mais recentes demostram que os esquemas com talidomida \pm dexametasona resultam em taxas de resposta ainda maiores do que dexametasona isoladamente ou VAD (até $70 \%$ em casos recém-diagnosticados). ${ }^{10,11}$ Outra vantagem do uso de talidomida \pm dexametasona é a garantia de mobilização adequada para transplante autólogo após quatro a seis meses do uso do esquema. Porém, pacientes tratados com esses protocolos apresentam maior risco de efeitos colaterais que em geral respondem à redução de dose: constipação intestinal, sedação, rash cutâneo e fadiga.

Entre os efeitos adversos mais graves secundários ao uso prolongado da talidomida estão a neuropatia periférica (que pode melhorar com a redução ou descontinuação da droga) e maior risco de fenômenos tromboembólicos, o que torna necessário o uso de profilaxia com heparina de baixo peso molecular, principalmente se a talidomida estiver sen- 
Quadro 1. Esquemas de tratamento de primeira linha para pacientes com MM candidatos à consolidação com altas doses de quimioterapia seguidas de transplante de células-tronco hematopoéticas

\begin{tabular}{|c|c|c|}
\hline Esquema & Doses & $\begin{array}{l}\text { Taxa de resposta } \\
\text { nos novos casos }\end{array}$ \\
\hline Dexametasona & $\begin{array}{l}\text { Repetir a cada } 4-5 \text { semanas } \\
\text { Dexametasona } 40 \text { mg VO D1-4, 9-12, 17-20 }\end{array}$ & $45 \%$ \\
\hline VAD & $\begin{array}{l}\text { Repetir a cada } 4 \text { semanas } 4 \text { a } 6 \text { ciclos } \\
\text { Vincristina } 0,4 \mathrm{mg} \text { EV IC D1-4 } \\
\text { Doxorubicina } 9 \mathrm{mg} / \mathrm{m}^{2} \text { IC D1-4 } \\
\text { Dexametasona } 40 \mathrm{mg} \text { VO D1-4, } 9-12,17-20\end{array}$ & $55-65 \%$ \\
\hline & $\begin{array}{l}\text { Repetir a cada } 4 \text { semanas } 4 \text { a } 6 \text { ciclos } \\
\text { Vincristina } 0,4 \mathrm{mg} \mathrm{EV} 30 \mathrm{~min} \text { D } 1-4 \\
\text { Doxorubicina } 9 \mathrm{mg} / \mathrm{m}^{2} 30 \mathrm{~min} \text { D } 1-4\end{array}$ & \\
\hline $\begin{array}{l}\text { VAD } \\
\text { ambulatorial }\end{array}$ & $\begin{array}{l}\text { Dexametasona } 40 \mathrm{mg} \text { VO D1-4, 9-12, 17-20 } \\
\text { ciclos ímpares }(1,3,5) \\
\text { Dexametasona } 40 \mathrm{mg} \text { VO D1-4 } \\
\text { ciclos pares }(2,4,6)\end{array}$ & $67 \%$ \\
\hline Talidomida & $200-400$ mg/dia VO D1-28 & $25 \%$ \\
\hline Tal/Dex & $\begin{array}{l}\text { Repetir a cada } 4 \text { semanas } \\
\text { Talidomida } 200 \mathrm{mg} / \mathrm{dia} \text { VO D 1-28 } \\
\text { Dexametasona } 40 \mathrm{mg} \text { VO D1-4, 9-12, 17-20 } \\
\text { (ciclos pares) e D1-4 (ciclos ímpares) }\end{array}$ & $65-70 \%$ \\
\hline
\end{tabular}

VO = via oral; IC = infusão contínua

Quadro 2. Esquemas de tratamento de primeira linha para pacientes com MM não-candidatos a transplante de células-tronco hematopoéticas

\begin{tabular}{|c|c|c|}
\hline Esquema & Doses & $\begin{array}{c}\text { Taxa de resposta } \\
\text { nos casos novos }\end{array}$ \\
\hline $\begin{array}{c}\text { Melfalano/ } \\
\text { prednisona (MP)* }\end{array}$ & $\begin{array}{l}\text { Repetir a cada } 6 \text { semanas até que a } \\
\text { doença atinja plateau (em geral, } 6 \text { ciclos) } \\
\text { Melfalano } 8-10 \text { mg VO D1-7 } \\
\text { Prednisona } 60 \mathrm{mg} \text { D1-7 }\end{array}$ & $50-55 \%$ \\
\hline Talidomida & $200-400$ mg/dia VO D 1-28 & $25 \%$ \\
\hline $\begin{array}{c}\text { Talidomida/ } \\
\text { Dexametasona }\end{array}$ & $\begin{array}{l}\text { Repetir a cada } 4 \text { semanas } \\
\text { Talidomida } 200 \mathrm{mg} / \mathrm{dia} \text { VO D 1-28 } \\
\text { Dexametasona } 40 \mathrm{mg} \text { VO D1-4, 9-12, 17-20 } \\
\text { (ciclos pares) e D1-4 (ciclos ímpares) }\end{array}$ & $65-70 \%$ \\
\hline $\begin{array}{c}\text { Melfalano/ } \\
\text { Prednisona/ } \\
\text { Talidomida (MPT) }\end{array}$ & $\begin{array}{l}\text { Repetir a cada } 4 \text { semanas, por } 6 \text { meses } \\
\text { Melfalano } 4 \mathrm{mg} / \mathrm{m}^{2} \text { VO D } 1-7 \\
\text { Prednisona } 40 \mathrm{mg} / \mathrm{m}^{2} \text { VO D } 1-7 \\
\text { Talidomida } 100 \mathrm{mg} / \mathrm{dia} \text { VO D 1-28 } \\
\text { Manutenção: Talidomida } 100 \mathrm{mg} / \mathrm{dia} \text { VO } \\
\text { até progressão }\end{array}$ & $76 \%$ \\
\hline
\end{tabular}

*Doses alternativas: melfalano $8 \mathrm{mg} / \mathrm{m}^{2} / \mathrm{dia} / 4$ dias e prednisona $100 \mathrm{mg} / \mathrm{dia}$ por 4 dias

\section{Pacientes com mais de 65-70 anos, com performance status ruim, comorbidades, com função renal alterada}

Pacientes não candidatos a transplante autólogo, pela idade ou comorbidades, podem receber tratamento com agentes alquilantes. O principal exemplo nessa categoria é o esquema melfalano e prednisona (MP). Esse esquema apresenta as vantagens de ser administrado por via oral, em paciente ambulatorial e ser bem tolerado. O tratamento é utilizado a cada seis semanas, até que a doença atinja plateau, o que ocorre em geral após seis ciclos de terapia. O principal objetivo do tratamento é atingir resposta com mínima toxicidade (Quadro 2). É importante lembrar que: 1) a dose do melfalano deve ser aumentada até que o tratamento seja capaz de produzir moderada citopenia no meio do ciclo de seis semanas (ao redor de três semanas após o início da terapia); 2) os pacientes com insuficiência renal devem receber dose reduzida de melfalano (50\% da dose se clearence creatinina $<40-50 \mathrm{ml} /$ min). O melfalano/prednisona (MP) é capaz de produzir taxas de resposta entre $50 \%-55 \% .^{13}$

Alternativamente, esquemas de poliquimioterapia como VAD (vincristina, doxorubicina e dexametasona) ou de dexametasona \pm talidomida podem ser empregados quando há necessidade de resposta mais rápida (doença óssea extensa e dolorosa, função renal com rápida deterioração).

Recentemente, Palumbo et a ${ }^{14}$ divulgaram os resultados do estudo fase III randomizado envolvendo 331 pacientes de 60 a 85 anos de idade, com MM recém-diagnosticado e que receberam melfalano/prednisona/talidomida (MPT) ou melfalano/ prednisona (MP). O esquema MPT atingiu taxas de resposta de $76 \%$ (15,5\% de resposta completa) versus $47,6 \%$ do MP (2,4\% de

do usada como terapia inicial e em combinação com dexametasona.

Devido a esses dois últimos efeitos colaterais potencialmente graves, alguns grupos como o Nordic Myeloma Study Group e o British Commitee for Standards in Haematology recomendam que a talidomida e combinações não devam ser usadas em MM recém-diagnosticados, exceto se no contexto de estudos clínicos. ${ }^{12}$ resposta completa). A toxicidade graus 3-4 no grupo MPT foi significativamente maior do que no grupo tratado com MP ( $48 \%$ e $25 \%$, respectivamente, $p=0,0002$ ). Os principais efeitos colaterais graves no grupo MPT foram: toxicidade hematológica (29 pacientes), tromboembolismo (13), infecções (12), neuropatia periférica (10). Durante o estudo, a introdução de heparina de baixo peso molecular reduziu o risco de tromboembolismo de $20 \%$ para $3 \%(p=0,005)$. A sobrevida 
livre de eventos (SLE) em dois anos com o MPT foi de 54\% e com o MP foi $27 \%$ ( $\mathrm{p}=0,0006)$. Porém, a sobrevida geral (SG) em três anos com o MPT foi de $80 \%$ e com o MP, $64 \%$ $(\mathrm{p}=0,19)$.

Outro estudo randomizado fase III comparando MP e MPT foi realizado pelo Intergroupe Francophone du Myélome (IFM) ${ }^{15}$ incorporando um terceiro braço de terapia de indução com quimioterapia habitual, mobilização de células progenitoras e dose intermediária de melfalano (100 $\mathrm{mg} / \mathrm{m}^{2}$ ou Mel 100) seguida de resgate com as células-tronco hematopoéticas. Nesse estudo, MPT foi associado a maior risco de toxicidade graus 3-4 (infecções, trombocitopenia, complicações tromboembólicas, neuropatia periférica, constipação e eventos cardíacos). Porém, os pacientes que receberam MPT apresentaram menor incidência de anemia, neutropenia, infecções, eventos cardíacos e óbitos nos primeiros três meses do que o grupo que recebeu dose intermediária de melfalano. Como no estudo de Palumbo et $a l,{ }^{14}$ aqui o MPT também apresentou maior incidência de resposta global quando comparado ao MP. Nesse estudo, a SLE e a SG do grupo tratado com o MPT foi superior ao $\mathrm{MP}(\mathrm{p}<0,001 \mathrm{e} \mathrm{p}=0,001$, respectivamente). Além disso, 0 MPT induziu maior SLE e SG do que a abordagem com Mel 100 ( $p=0,001$ e $p=0,004$, respectivamente). Baseado nesses resultados, Orlowski ${ }^{16}$ acredita o MPT possa ser considerado como o padrão-ouro para tratamento inicial de pacientes idosos e sintomáticos com MM. Lenalidomida (Revlimid), um novo agente imunomodulatório mais potente e menos tóxico do que a talidomida, poderá ser uma futura opção a essa última no MPT.

Talidomida/ciclofosfamida/dexametasona (Tha/Cy/ Dex) é outra potencial combinação para pacientes idosos recém-diagnosticados com MM. Recentemente, foi testada em 71 pacientes com MM refratários/recidivados. ${ }^{17} \mathrm{O}$ esquema garantiu $71 \%$ de resposta global, com $10 \%$ de respostas completas após seis meses de terapia, $66 \%$ de SG e $57 \%$ de SLE em dois anos. Entre os efeitos adversos graus 3-4, houve 7\% de eventos tromboembólicos (não foi realizada profilaxia).

Um estudo randomizado fase III foi coordenado pelo Eastern Cooperative Oncology Group utilizando talidomida/ dexametasona (Tal/Dex) ou dexametasona (Dex) isoladamente em 207 casos de MM recém-diagnosticados. ${ }^{18} \mathrm{~A}$ taxa de resposta a Tal/Dex foi de 63\% versus $41 \%$ no grupo Dex (p= $0,0017)$. Quanto aos efeitos adversos graus 3-4 nos primeiros quatro meses do estudo, Tal/Dex superou Dex (45\% versus $21 \%)(p<0,001)$.

Outra possibilidade terapêutica para tratamento de primeira linha em pacientes não-candidatos à consolidação com altas doses de quimioterapia seguidas de transplante de células-tronco hematopoéticas inclui o inibidor de proteassoma bortezomibe. VMP(Velcade, melfalano, prednisona) é uma opção para tratamento de pacientes com deleção do cromosssomo 13 (del 13), documentada por citogenética con- vencional ou FISH, pois nesses casos o bortezomibe parece superar o prognóstico desfavorável relacionado à alteração cromossômica. ${ }^{16}$

\section{Recomendações}

- Até o momento, não existem evidências de que pacientes com MM assintomático devam receber tratamento.

- Para o paciente sintomático (anemia, hipercalcemia, alteração da função renal, presença de lesões líticas ou plasmocitoma extramedular, aumento progressivo do componente-M no soro e/ou urina), o tratamento deverá ser rapidamente instituído. Os pacientes sintomáticos devem ser divididos em dois grupos: aqueles que são considerados candidatos à consolidação com altas doses de quimioterapia seguidas de transplante de células-tronco hematopoéticas e aqueles não elegíveis para receber altas doses de quimioterapia.

- Pacientes com menos de 65-70 anos, com bom performance status, sem comorbidades, com função renal próxima do normal: terapia de indução por 4 a 6 ciclos sem alquilante (evitando prejuízo na mobilização de células-tronco): blocos de dexametasona, VAD ou talidomida \pm dexametasona.

- Pacientes com mais de 65-70 anos, com performance status ruim, comorbidades: esquemas baseados em melfalano (MP, MPT) ou talidomida (Tha/Cy/Dex, Tal/Dex).

- Importante: Os pacientes com insuficiência renal devem receber dose reduzida de melfalano (50\% da dose se clearence cratinina $<40-50 \mathrm{ml} / \mathrm{min}$ ).

\section{Abstract}

The aim of this review is to evaluate the current status of first line treatment in multiple myeloma. Asymptomatic patients should not receive treatment at diagnosis. However, symptomatic patients (anemia, hypercalcemia, deterioration in renal function, lytic lesions or extramedullary plasmacytomas, increase of M-component in serum or urine) should receive treatment as soon as possible. Patients younger than 65-70 years old with good performance status, no concurrent diseases and normal renal function are possible candidates for consolidation using high-dose chemotherapy followed by stem-cell transplantation. Therefore, they should not receive drugs that could potentially interfere with stem-cell mobilization (such as alkylating agents). Thus, their treatment options are: dexamethasone, VAD and thalidomide (with or without dexamethasone). Thalidomide should be preferentially used in the context of clinical trials due to increased risks of neuropathy and thrombosis. Patients older than 65-70 years old and those with bad performance status, concurrent diseases or deterioration of renal function can receive alkylating 
agents (such as melphalan/prednisone) because they are not candidates for stem cell transplantation. The aim of their treatment is to reach a disease plateau with low toxicity rates. Other alkylating agents, thalidomide and corticosteroid combinations have higher response rates than melphalan/prednisone however, these combinations present more side effects and do not increase overall survival. Rev. bras. hematol. hemoter. 2007;29(1):31-35.

Key words: Multiple myeloma; treatment; chemotherapy.

\section{Referências Bibliográficas}

1. Kyle RA, Rajkumar SV. Multiple myeloma. N Engl J Med 2004; 351(18): 1860-73.

2. International Myeloma Working Group. Criteria for the classification of monoclonal gammopathies, multiple myeloma and related disorders: a report of the International Myeloma Group. Br J Haematol 2003;121:749-57.

3. Reece DE. An update of the management of multiple myeloma: the changing landscape, Hematology 2005:353-9.

4. Alexanian R, Barlogie B, Tucker S. VAD-based regimens as primary treatment for multiple myeloma. Am J Hematol 1990; 33:86-9.

5. Alexanian R, Barlogie B, Dixon D. High-dose glucocorticoid treatment of resistant myeloma. Ann Intern Med 1986; 105:8-11.

6. Cavo M, Galieni P, Tassi C, Gobbi M, Tura S. M-2 protocol for melphalan-resistant and relapsing multiple myeloma. Eur J Haematol 1988;40:168-173.

7. Alexanian R, Barlogie B, Ventura G. Chemotherapy for resistant and relapsing multiple myeloma. Eur J Haematol Suppl 1989; 51:140-4.

8. Alexanian R, Dimopoulos MA, Delasalle K, Barlogie B. Primary dexamethasone treatment of multiple myeloma. Blood 1992; 80: 887-90.

9. Segeren CM, Sonneveld P, van der Holt B, Baars JW, Biesma DH, Cornellissen JJ, et al. Vincristine, doxorubicin and dexametasone (VAD) administered as rapid intravenous infusion for first-line treatment in untreated multiple myeloma. Br J Haematol 1999; 105:127-30.

10. Rajkumar SV, Hayman S, Gertz MA. Combination therapy with thalidomide plus dexamethasone for newly diagnosed myeloma. J Clin Oncol 2002;20:4.319-23.

11. Cavo M, Zamagni E, Tosi P, Tacchetti P, Cellini C, Cangini D, et al; Bologna 2002 study. Superiority of thalidomide and dexamethasone over vincristine-doxorubicindexamethasone (VAD) as primary therapy in preparation for autologous transplantation for multiple myeloma. Blood 2005; 106(1):35-9.

12. Smith A, Wisloff F, Samson D, UK Myeloma Forum, Nordic Myeloma Study Group and British Committee for Standards in Haematology. Guidelines on the diagnosis and management of multiple myeloma 2005. Br J Haematol 2005;132:410-51.

13. Myeloma Trialists' Collaborative Group. Combination chemotherapy versus melphalan plus prednisone as treatment for multiple myeloma: an overview of 6,633 patients from 27 randomized trials. J Clin Oncol 1998;16:3832-42.

14. Palumbo A, Bringhen S, Caravita T, Merla E, Capparella V, Callea $\mathrm{V}$, et al; Italian Multiple Myeloma Network, GIMEMA. Oral melphalan and prednisone chemotherapy plus thalidomide compared with melphalan and prednisone alone in elderly patients with multiple myeloma: randomised controlled trial. Lancet. 2006; 367(9513):825-31.
15. Facon T, Mary J, Harousseau J, et al. Superiority of melphalanprednisone (MP)+thalidomide (THAL) over MP and autologous stem cell transplantation in the treatment of newly diagnosed elderly patients with multiple myeloma. J Clin Oncol 2006:24:1s.

16. Orlowski RZ. Initial therapy of multiple myeloma patients who are not candidates for stem cell transplantation. Hematology 2006; 338-347.

17. Garcia-Sanz R, Gonzalez-Porras JR, Hernandez JM, Polo-Zarzuela M, Sureda A, Barrenetxea C, et al. The oral combination of thalidomide, cyclophosphamide and dexamethasone (ThaCyDex) is effective in relapsed/refractory multiple myeloma. Leukemia. 2004;18(4):856-63.

18. Rajkumar SV, Blood E, Vesole DH, Fonseca R, Greipp PR. Phase III clinical trial of thalidomide plus dexamethasone compared with dexamethasone alone in newly diagnosed multiple myeloma: a clinical trial coordinated by the Eastern Cooperative Oncology Group. J Clin Oncol 2006;24:431-6.

O tema apresentado e o convite ao(s) autor(es) consta da pauta elaborada pelo co-editor.

Avaliação: Co-editor e um revisor externo.

Publicado após revisão e concordância do editor.

Conflito de interesse: não declarado.

Recebido: 25/11/2006

Aceito: 05/01/2007 